\title{
Identification of a Biomarker Panel for Early Detection of Lung Cancer Patients.
}

\author{
Bethany Geary ${ }^{1,2}$, Michael J. Walker ${ }^{2}$, Joseph T. Snow ${ }^{2,3}$, David C. H. Lee ${ }^{1}$, Maria \\ Pernemalm ${ }^{4}$, Saeedeh Maleki-Dizaji ${ }^{2}$, Narges Azadbakht ${ }^{2}$, Sophia Apostolidou ${ }^{5}$, Julie \\ Barnes $^{6}$, Piotr Krysiak ${ }^{7}$, Rajesh Shah7 ${ }^{7}$ Richard Booton ${ }^{8}$, Caroline Dive ${ }^{9,10}$, Philip A \\ Crosbie ${ }^{1,5,8}$, Anthony D. Whetton ${ }^{1,3,10,}{ }^{*}$. \\ ${ }^{1}$ Stoller Biomarker Discovery Centre, Institute of Cancer Sciences, Faculty of Medical and Human \\ Sciences, University of Manchester, Manchester, United Kingdom \\ ${ }^{2}$ Stem cell and leukaemia proteomics laboratory, Institute of Cancer Sciences, Faculty of Medical and \\ Human Sciences, University of Manchester, Manchester, United Kingdom \\ ${ }^{3}$ Department of Earth Sciences, University of Oxford, Oxford, United Kingdom. \\ ${ }^{4}$ Science for Life Laboratory, Department of Oncology and Pathology, Karolinska Institutet, Stockholm, \\ Sweden. \\ ${ }^{5}$ Gynaecological Cancer Research Centre, Department of Women's Cancer, Institute for Women's Health, \\ University College London, London, United Kingdom \\ ${ }^{6}$ Abcodia, Cambourne, Cambridgeshire, United Kingdom \\ 7 Department of Thoracic Surgery, Wythenshawe Hospital, Manchester University NHS Foundation Trust, \\ United Kingdom \\ ${ }^{8}$ North West Lung Centre, Wythenshawe Hospital, Manchester University NHS Foundation Trust, United \\ Kingdom \\ ${ }^{9}$ Clinical and Experimental Pharmacology Group, Cancer Research UK Manchester Institute, University of \\ Manchester, Manchester, United Kingdom \\ ${ }^{10}$ Cancer Research UK Lung Cancer Centre of Excellence
}

* Corresponding author: Anthony D. Whetton tony.whetton@manchester.ac.uk +44 (0)161 2756267

\section{Contents}

\section{Supplemental tables}

Contents

Table S1. IPA analysis of proteins with higher abundance in the cancer draining vein compared to the non-cancer draining vein.

Table S2. IPA analysis of proteins with higher abundance in the cancer draining vein compared to the artery.

Table S3. IPA analysis of proteins with significant differential abundance in patients within 1 year of cancer diagnosis. 
Table S1. IPA analysis of proteins with higher abundance in the cancer draining vein compared to the non-cancer draining vein.

\begin{tabular}{|l|c|}
\hline Associated network functions & $\begin{array}{c}\text { Number of } \\
\text { associated } \\
\text { proteins }\end{array}$ \\
\hline Cardiovascular System Development and Function, Cellular Movement, Cancer & 26 \\
\hline Lipid Metabolism, Small Molecule Biochemistry, Cardiovascular Disease & 22 \\
\hline Cellular Movement, Cellular Development, Tissue Development & 17 \\
\hline Cancer, Cardiovascular Disease, Cell Death and Survival & 2 \\
\hline Cardiovascular Disease, Cell Death and Survival, Connective Tissue Disorders & 2 \\
\hline & \\
\hline Top diseases and disorders & p value \\
\hline Cancer & 0.048 \\
\hline Organismal injury and abnormalities & 0.048 \\
\hline Inflammatory disease & 0.030 \\
\hline Respiratory disease & 0.048 \\
\hline Endocrine system disorders & 0.048 \\
\hline
\end{tabular}

Table S2. IPA analysis of proteins with higher abundance in the cancer draining vein compared to the artery.

\begin{tabular}{|l|c|}
\hline Associated network functions & $\begin{array}{c}\text { Number of } \\
\text { associated } \\
\text { proteins }\end{array}$ \\
\hline RNA Damage and Repair, Inflammatory Response, Cell Cycle & 20 \\
\hline Inflammatory Response, Organismal Injury and Abnormalities, Cell Death and Survival & 15 \\
\hline $\begin{array}{l}\text { Cardiovascular System Development and Function, Embryonic Development, } \\
\text { Nervous System Development and Function }\end{array}$ & 2 \\
\hline Cancer, Cell Death and Survival, Cellular Development & 2 \\
\hline Cellular Movement, Hematological System Development and Function, Cancer & 2 \\
\hline Top diseases and disorders & $\mathbf{p ~ v a l u e ~}$ \\
\hline Cancer & 0.049 \\
\hline Cardiovascular disease & 0.037 \\
\hline Connective tissue disorders & 0.038 \\
\hline Dermatological diseases and conditions & 0.049 \\
\hline Endocrine system disorders & 0.043 \\
\hline
\end{tabular}


Table S3. IPA analysis of proteins with significant differential abundance in patients within 1 year of cancer diagnosis

Organismal Survival, Dermatological Diseases and Conditions, Organismal Injury and 37

Abnormalities

Cellular Movement, Cellular Development, Cellular Growth and Proliferation

Cellular Growth and Proliferation, Cell Cycle, Cellular Movement

Cancer, Organismal Injury and Abnormalities, Cell Death and Survival

RNA Post-Transcriptional Modification, Connective Tissue Disorders, Developmental

Disorder

Top diseases and disorders

p value

Organismal injury and abnormalities

0.006

Gastrointestinal disease

0.013

Connective tissue disorders

0.014

Dermatological diseases and conditions 0.014

Developmental disorder

0.014

